\title{
A Dynamic Model coupling Photoacclimation and Photoinhibition in Microalgae
}

\author{
Philipp Hartmann ${ }^{1}$, Andreas Nikolaou ${ }^{2}$, Benoît Chachuat $^{2}$, Olivier Bernard ${ }^{1}$
}

\begin{abstract}
Microalgae are often considered a promising alternative for production of renewable energy, particularly as a potential producer of biodiesel. In order to improve largescale, industrial culturing systems, the development of mathematical models that are capable of predicting photosynthetic productivity under dynamic conditions is crucial. Especially important are the processes of growth inhibition due to excess light, known as photoinhibition, and of adjustment of the light harvesting capacity to photon flux, known as photoacclimation. In this paper, we develop a dynamic model that accounts for the processes of photoinhibition, photoacclimation and growth in microalgae, thereby spanning multiple time scales. The properties of the model are investigated under quasi steadystate conditions and the model is validated against several experimental data sets from the literature. We also discuss how the model can provide new insights into the mechanisms underlying photoacclimation.
\end{abstract}

\section{INTRODUCTION}

Algal-derived biofuel has been considered a potential alternative source of renewable energy since the 1970s [1]. The claimed advantages of this approach are a high actual photosynthetic yield compared to field crops, an independence to agriculturally usable soil, and a possibility to avoid fresh water consumption [2]. These advantages could lead to large-scale production of algal biomass which is not in direct competition with food production. Moreover, microalgae can contribute to $\mathrm{CO}_{2}$ mitigation due to their inherent consumption of $\mathrm{CO}_{2}$ during photosynthesis, they can be coupled with wastewater treatment technologies [3], and they can produce high added-value products such as cosmetics, pharmaceuticals and nutraceuticals [4]. All these possibilities have put microalgae in a favorable position for their integration into biorefinery concepts. Nonetheless, many problems have yet to be overcome on the path to making large-scale production of microalgal biofuel feasible.

For over a decade, much research effort has been devoted to enhancing lipid production, from which biodiesel can be derived. Lipid productivity can be stimulated by a deprivation of nutrients (nitrogen or phosphorus) as well as by a modification of the light conditions. It has been reported that microalgae can reach a high lipid content (over $50 \%$ of dry weight) following a nitrogen limitation [5], and also that alternating the light intensity and varying the light/dark

${ }^{1}$ BIOCORE-INRIA, BP93, 06902 Sophia-Antipolis Cedex, France and UPMC-CNRS LOV, BP28, 06234 Villefranche sur mer, France (e-mail: \{philipp.hartmann, olivier.bernard\} @inria.fr)

${ }^{2}$ Center for Process Systems Engineering, Department of Chemical Engineering, Imperial College London, South Kensington Campus, London SW7 2AZ, UK (e-mail: \{andreas.nikolaou10, b.chachuat eimperial.ac.uk) periods can have important effects in terms of microalgae growth and lipid accumulation [6].

Two key processes are involved in the way light conditions and nutrient supply affect the photosynthetic yield. Photoinhibition causes a loss of photosynthetic yield due to an excess of irradiance which damages some of the key proteins in the photosynthetic apparatus. Photoacclimation, the process by which microalgae adapt their pigment composition to the light intensity, also alters the rate of photosynthesis production. These two processes operate at different time scales and are tightly coupled. Photoinhibition occurs in a time scale of minutes, whereas photoacclimation acts over a time scale of days. In order to achieve an optimal microalgae productivity, understanding the processes of nutrient assimilation, photoinhibition and photoacclimation, as well as their interactions, is therefore paramount. A number of mathematical models are available that account for photoacclimation in the slow time scale [7], [8], yet they neglect the photoinhibition processes. On the other hand, models have also been proposed which describe photoinhibition in the fast time scale [9], but they do not account for photoacclimation.

It is precisely the objective of this paper to develop a dynamic model of microalgae growth that couples photoinhibition and photoacclimation with carbon and nitrogen uptake. Nutrient assimilation is described by the well accepted and validated Droop model [10]. Photoinhibition is described by a model proposed by Han [9], originating in the work of Eilers and Peeters [11] who introduced the concept of photosynthetic factories (or photosynthetic units). Photoacclimation effects are incorporated in the expressions of photosynthesis rate and pigment synthesis rate using empirical relations [12]. This last part constitutes the main novelty of the proposed model. More specifically, the effective cross section and the number of photosynthetic units-which are parameters in the Han model-are expressed as functions of the chlorophyll content. This approach leads to a simple expression for the photosynthesis rate, which conveniently lends itself to mathematical analysis.

The remainder of the paper is organized as follows. The derivation of the coupled model is presented first. Models for processes occurring at the slow and fast time scales are reviewed in Sect. II and Sect. III, respectively. The former includes both growth and photoacclimation processes, while the latter is concerned with photoinhibition. In Sect. IV, an extension of the photosynthesis rate (or growth rate) expression is presented, which incorporates a dependence to the state of the photosynthetic units via the chlorophyll content. Properties of the resulting PI response curve are 
also analyzed and empirical expressions are proposed for the functional cross section and the number of photosynthetic units. A preliminary calibration and validation of the coupled model based on several sets of experimental data from the literature is presented in Sect. V. Finally, conclusions are drawn in Sect. VI and future research directions are discussed.

\section{Modelling of Slow Time-Scale Processes}

\section{A. Nutrient-Limited Growth}

Since the 1970s, it has been observed that microalgae keep growing for a long time after nutrients are depleted. This apparent decoupling between nutrient uptake and growth processes can be explained by the fact that the entire metabolic pathway from nutrient assimilation to protein synthesis involves numerous steps, some of which are time consuming [13]. In this context, Monod kinetics are no longer appropriate for predicting microalgae growth. A better way to represent nutrient-limited growth is by separating the nutrient uptake rate, denoted by $\rho$ hereafter, from the growth rate, denoted by $\mu$. This idea was followed by Droop [10], [14] in order to relate the growth rate to the internal nitrogen quota. Since its introduction, the Droop model has been widely studied [15]-[17] and thoroughly validated [10], [17]-[19].

Recently, the Droop model has been extended to account for the effect of light conditions [8]. The growth rate $\mu$ is expressed as

$$
\mu(q, \cdot)=\bar{\mu}\left(1-\frac{Q_{0}}{q}\right) \phi(\cdot),
$$

where $\bar{\mu}$ stands for the maximum growth rate, that is, the growth rate reached under non-limiting conditions; $q$, the carbon-specific nitrogen quota of the cells (in units of $\mathrm{g}_{\mathrm{N}} / \mathrm{g}_{\mathrm{C}}$ ); and $Q_{0}$, the minimal cell quota. In particular, the growth rate is equal to 0 at a quota of $q=Q_{0}$. Later in Sect. IV, an expression of the saturation function $\phi(\cdot)$ will be developed that accounts for the state of the photosystem units.

The nutrient uptake rate $\rho$, on the other hand, can be expressed as [8], [20]

$$
\rho(s, q)=\bar{\rho} \frac{s}{s+k_{s}}\left(1-\frac{q}{Q_{l}}\right),
$$

where $s$ stands for the nutrient (inorganic nitrogen) concentration in the bulk phase; $\bar{\rho}$, the maximum nutrient uptake rate; and $k_{s}$, the half-saturation constant for substrate uptake. Moreover, the term $\left(1-q / Q_{l}\right)$ in (2) constrains the internal quota $q$ as

$$
Q_{0} \leq q \leq Q_{l}
$$

This way $Q_{l}$ defines the maximum cell quota, which is obtained in nutrient-replete conditions.

Using the expressions for $\rho$ and $\mu$ in (1) and (2), the mass balances for $s, q$, and the biomass concentration $x$ in a CSTR can be expressed as

$$
\begin{aligned}
\dot{s} & =D s_{\text {in }}-\rho(s, q) x-D s \\
\dot{q} & =\rho(s, q)-\mu(q, \cdot) q \\
\dot{x} & =\mu(q, \cdot) x-D x-R x,
\end{aligned}
$$

with $D$ and $R$ denoting, respectively, the dilution rate and the respiration rate; and $s_{\text {in }}$, the nutrient concentration in the feed.

\section{B. Pigment Content}

Photoacclimation refers to adaptation mechanisms taking place on a time scale of days, by which the chlorophyll content and the pigment composition change in response to variations in the light irradiance. It has been suggested that microalgae use photoacclimation as a means to optimize their growth at low irradiance and to minimize damage at high irradiance [12].

One way to describe photoacclimation is by accounting for the change in the chlorophyll content over time. As a first approximation, the chlorophyll content can be assumed to be proportional to cellular protein content, which is itself represented by the particulate nitrogen content $x q$ [8]:

$$
\mathrm{Chl}=\gamma\left(I_{g}\right) x q
$$

where $I_{g}$ represents the light irradiance at which the cells are acclimated, also called growth irradiance. The saturation function $\gamma$ is given by

$$
\gamma\left(I_{g}\right)=\bar{\gamma} \frac{k_{I}}{I_{g}+k_{I}},
$$

with saturation parameters $\bar{\gamma}$ and $k_{I}$. Moreover, the dynamics of $I_{g}$ can be described by the following equation

$$
\dot{I}_{g}=\delta \mu(q, \cdot)\left(I-I_{g}\right)
$$

where $I$ stands for the current light irradiance. This equation assumes that the adaptation speed is proportional to the defect $\left(I-I_{g}\right)$ as well as the current growth rate $\mu$, with a proportionality parameter $\delta$. Overall, a change in the current irradiance $I$ will affect $I_{g}$ via (6), which in turn will modify the chlorophyll content Chl via (4) and (5).

A simplified expression of the chlorophyll dynamics, which removes $I_{g}$ as an intermediate variable, is as follows:

$$
\mathrm{Chl}=\delta \mu(q, \cdot)(\gamma(I) x q-\mathrm{Chl}),
$$

Although not equivalent to (4)-(6) from a strict mathematical viewpoint, these two models share the same steady states. Moreover, the simplified model (7) is appealing in that it features linear dynamics and it removes variable $I_{g}$ which cannot be measured directly.

Let $\theta$ represent the carbon-specific chlorophyll quota (in units of $\left.\mathrm{g}_{\mathrm{Chl}} / \mathrm{g}_{\mathrm{C}}\right)$, defined as $\theta x=\mathrm{Chl}$. We can in turn define the rate of carbon uptake per chlorophyll unit, $\mu_{\mathrm{Chl}}$, so that

$$
\theta \mu_{\mathrm{Chl}}(q, \cdot)=\mu(q, \cdot) .
$$

$\mu_{\mathrm{Chl}}$ is often referred to as the chlorophyll-specific photosynthesis rate. 


\section{Modelling of FAst Time-Scale Processes}

The Han model [9] describes the effect of light irradiance on microalgae growth, including photoinhibition via the damaging of key proteins in the photosynthetic units, which is considered the main limiting factor. Perhaps the main merit of the Han model lies in the reduction of this complex photosynthesis process into three possible states of the photosystem II (PSII) unit. These states are open (A), closed (B) or inhibited (C).

- Photosynthetic production is described by the transitions between open and closed states, that is between A and B. Excitation is assumed to occur at a rate of $\sigma I$, with $\sigma$ being the functional cross section of the photosynthetic unit (PSU), while deexcitation is assumed to occur at a rate of $\frac{1}{\tau}$, with $\tau$ the turnover time of the electron transport chain.

- Photoinhibition occurring at high light irradiance corresponds to the transition from closed to inhibited state, that is from B to $\mathrm{C}$. This process is assumed to occur at a rate of $k_{d} \sigma I$. The reverse transition between states $\mathrm{C}$ and $\mathrm{B}$ describes the repair of inhibited PSUs by enzymatic processes in the cell, a mechanism that is assumed to occur at a constant rate $k_{r}$.

The equations describing the rates of change in the fractions of open, closed and inhibited states are given by:

$$
\begin{aligned}
\dot{A} & =-I \sigma A+\frac{B}{\tau} \\
\dot{B} & =I \sigma A-\frac{B}{\tau}+k_{r} C-k_{d} \sigma I B \\
\dot{C} & =-k_{r} C+k_{d} \sigma I B .
\end{aligned}
$$

The variables $A, B$ and $C$ denote the fractions of PSUs in open, closed and inhibited states, respectively. Note that these fractions will sum up to 1 at any time.

Interestingly, expressions giving the fractions of open, closed and inhibited states at steady state as a function of the irradiance $I$ can be derived explicitly from (9). The steadystate expression $A^{\infty}$ for the open state $\mathrm{A}$ is given by

$$
A^{\infty}(I)=\frac{1}{1+\tau \sigma I+K \tau \sigma^{2} I^{2}},
$$

with $K:=k_{d} / k_{r}$.

\section{A Combined Model of Slow and Fast Time-SCALE Processes}

The proposed model couples three dynamic processes, namely (i) the dynamics of the PSUs, (ii) the dynamics of intracellular nitrogen content, and (iii) the dynamics of the chlorophyll content. These processes span four different timescales-the dynamics of the PSUs already spans two separate time scales-ranging from seconds for the openclosed dynamics of the PSUs up to several days for the dynamics of intracellular nitrogen quota $q$.

Our model builds upon the Droop-Han model by [21] and incorporates photoacclimation processes via the dynamics of the chlorophyll content as detailed in Sect. II-B. To make this coupling possible, the main idea is to include a dependence to the state of the PSUs in the expression of the growth rate $\mu$ in (1)—or, equivalently, in the expression of the growth rate $\mu_{\mathrm{Chl}}$ in (8).

- Note first that the probability that a photon encounters an open state is proportional to $I A$. Therefore, it is assumed here that the saturation term $\phi(\cdot)$ in (1) is itself proportional to $I A$.

- Falkowski [12] describes photoacclimation as a process that can follow either one of two strategies. The socalled $n$-strategy corresponds to a change in the density (or number) of PSUs, denoted by $N$ subsequently. The $s$-strategy corresponds to a change in the size of the PSU, and is thus directly related to the functional cross section $\sigma$. In practice, chlorophyll is either used to build new PSUs or to enrich already existing PSUs, and these two acclimation strategies occur concurrently. Both can be described by defining $N$ and $\sigma$ as functions of the chlorophyll quota $\theta$, and by assuming the saturation term $\phi(\cdot)$ to be proportional to both $N(\theta)$ and $\sigma(\theta)$.

Based on these considerations, the expression for the growth rate $\mu_{\mathrm{Chl}}$ can be rewritten as

$$
\mu_{\mathrm{Chl}}(q, \theta, I)=\bar{\alpha}\left(1-\frac{Q_{0}}{q}\right) N(\theta) \sigma(\theta) I A(I),
$$

with $\bar{\alpha}$ a constant parameter. In particular, the fraction $A$ of PSUs in open state can be replaced with its steadystate expression $A^{\infty}(I)$ in (10) when considering time scales slower than hours.

\section{A. Analysis of the PI Response}

Typical experiments for assessing the photosynthetic efficiency proceed by using microalgae previously photoacclimated to a light intensity $I_{g}$, and then subject them to a series of increasing light irradiance $I$. The instantaneous growth rate obtained under these conditions, either $\mu$ or $\mu_{\mathrm{Chl}}$, is then measured and yields the so-called PI response curve when plotted as a function of $I$.

An important characteristic of PI curves is their initial slope, i.e., the rate of change of $\mu_{\mathrm{Chl}}$ with respect to the light irradiance $I$, for vanishing values of the latter. An expression of this initial slope $\alpha$ can be obtained from (10) and (11) as

$$
\alpha(q, \theta)=\bar{\alpha}\left(1-\frac{Q_{0}}{q}\right) \sigma(\theta) N(\theta) .
$$

Regarding the dependence to the chlorophyll quota $\theta$, $\alpha$ is seen to be proportional to $\sigma(\theta) N(\theta)$. Many authors concur to say that, for many microalgae species, $\alpha(\theta)$ can be considered to be independent of the value of $\theta$ [22]. In other words, the term $\sigma(\theta) N(\theta)$ can be assumed to be constant, equal to $\sigma N^{\circ}$. Under these assumptions, the PI curve expression reduces to

$$
\mu_{\mathrm{Chl}}(q, \theta, I)=\bar{\mu}_{\mathrm{Chl}}(q) \frac{I}{1+\tau \sigma(\theta) I+K \tau \sigma(\theta)^{2} I^{2}}
$$

with $\bar{\mu}_{\mathrm{Chl}}(q):=\bar{\alpha}\left(1-\frac{Q_{0}}{q}\right) \sigma N^{\circ}$. Nonetheless, the constant initial slope assumption remains a subject of intense debate; 
see, for instance, the paper by Richardson et al. [23] where microalgae acclimation strategies are divided into six different categories based on photosynthesis-irradiance response data.

Concerning the dependence of $\alpha$ to the internal quota $q$, it can be assumed as a first approximation that the preacclimation period does not significantly affect $q$; that is, the variation of $\bar{\mu}_{\mathrm{Chl}}(q)$ during the (short) time of the PI curve experiment is neglected.

\section{B. Structural Properties of the PI Response Curves}

We start by analyzing the implications of the foregoing modeling assumptions in terms of the structure of the PI curve. The optimum irradiance value $I^{\star}$ maximizing $\mu_{\mathrm{Chl}}$ can be determined based on (13) as

$$
I^{\star}(\theta)=\frac{1}{\sigma(\theta) \sqrt{K \tau}}=\frac{N(\theta)}{\sigma N^{\circ} \sqrt{K \tau}} .
$$

In turn, it is not hard to see that the maximum productivity rate $\mu_{\mathrm{Chl}}^{\star}$ can be expressed as

$$
\mu_{\mathrm{Chl}}^{\star}(q, \theta)=\bar{\mu}_{\mathrm{Chl}}(q) \frac{\sigma N^{\circ} \sqrt{K \tau}}{\tau+2 \sqrt{K \tau}} I^{\star}(\theta) .
$$

A key property of the proposed model is therefore the linear relation between $I^{\star}$ and $\mu_{\mathrm{Chl}}^{\star}$, which is itself a direct consequence of the assumption that the initial slope of the PI curve is constant regardless of the acclimation state. In particular, this property is independent of particular choices of the acclimation functions $\sigma(\theta)$ and $N(\theta)$. Besides, this property can easily be tested using data from experimental PI curves obtained at different acclimation states.

The analysis can be taken one step further by postulating a particular relationship between $\sigma$ (or $N$ ) and the chlorophyll quota $\theta$. Here, power laws are selected to represent the dependence in $\theta$, giving

$$
\sigma(\theta)=\beta \theta^{\kappa}, \quad N(\theta)=\frac{\sigma N^{\circ}}{\beta} \theta^{-\kappa},
$$

with parameters $\beta, \kappa \geq 0$.

Besides simplicity, expressing $\sigma(\theta)$ as a power law function (16) also makes sense from a physical point of view. It is expected that $\sigma(\theta)$ will be monotonically increasing, as the size of the PSUs typically increases with an increasing chlorophyll quota $\theta$; that is, $\sigma^{\prime}(\theta)$ will be nonnegative. Moreover, due to the packaging effect in the PSUs [12], $\sigma^{\prime}(\theta)$ can be expected to be non-increasing. Accordingly, the value of the exponent $\kappa$ should be in the range $[0,1]$.

The final expression of the growth rate in the slow time scale reads

$$
\begin{aligned}
\mu_{\mathrm{Chl}}(q, \theta, I) & =\bar{\mu}_{\mathrm{Chl}}(q) \frac{I}{1+\tau \beta \theta^{\kappa} I+K \tau \beta^{2} \theta^{2 \kappa} I^{2}} \\
& =\bar{\alpha}\left(1-\frac{Q_{0}}{q}\right) \frac{\sigma N^{\circ} I}{1+\tau \beta \theta^{\kappa} I+K \tau \beta^{2} \theta^{2 \kappa} I^{2}} .
\end{aligned}
$$

\section{Preliminary Validation of the Coupled Model}

\section{A. Model Calibration based on Experimental Data from the Literature}

A preliminary calibration of the new features in the coupled model is conducted in this subsection. Several sets of experimental data from the works of Anning et al. [24] and Falkowski \& Owens [25] are considered. The emphasis is first on the chlorophyll-specific photosynthesis rate (17), and then on the chlorophyll content (4),(5).

1) Chlorophyll-Specific Photosynthesis Rate Expression: The values of the parameters $\beta, \kappa$ and $\bar{\mu}_{\mathrm{Chl}}$ in (17) are estimated using a nonlinear least-square minimization procedure (global optimization solver BARON in GAMS [26]). On the other hand, values for the Han model parameters $k_{r}, k_{d}$ and $\tau$ are taken from the literature [21]; these values are reported in Table I for the sake of reproducibility.

\begin{tabular}{cc}
\hline Parameter & Value \\
\hline$\tau$ & $6.850 \mathrm{~s}$ \\
$k_{r}$ & $4.80 \times 10^{-4} \mathrm{~s}^{-1}$ \\
$k_{d}$ & $2.99 \times 10^{-4}-$ \\
\hline \multicolumn{2}{c}{ TABLE I }
\end{tabular}

PARAMETER VALUES IN THE HAN MODEL.

- The experimental data by Anning et al. [24] are for the diatom Skeletonema costatum. They concern two acclimation states at different growth irradiances $I_{g}$, namely 50 mol photons $\mathrm{m}^{-2} \mathrm{~s}^{-1}$ (LL) and 1500 mol photons $\mathrm{m}^{-2} \mathrm{~s}^{-1}$ (HL). Moreover, the LL irradiance corresponds to a chlorophyll quota of $\theta=0.082 \mathrm{~g}_{\mathrm{Chl}} \mathrm{g}_{\mathrm{C}}^{-1}$, and the HL irradiance to $\theta=$ $0.018 \mathrm{~g}_{\mathrm{Chl}} \mathrm{g}_{\mathrm{C}}^{-1}$. The resulting fits in terms of the chlorophyll specific photosynthesis rate $\mu_{\mathrm{Chl}}$ are shown in Figure 1. The model predictions are in good agreement with both experimental data sets, thereby providing a first validation of the structural assumptions in (17). The estimated parameter values can be found in Table II. Note in particular the value of $\kappa=0.542$, which is in agreement with the considerations in Sect. IV-B.

- The experimental data by Falkowski \& Owens [25] are for the chlorophyte Dunaliela tertiolocta. Among the available data, four PI curves are selected, which were not affected by 'bleaching'. These curves are for growth irradiances $I_{g}$ of $60 \mu \mathrm{mol}$ photons $\mathrm{m}^{-2} \mathrm{~s}^{-1}$ $\left(\mathrm{LL}_{1}\right), \quad 120 \mu$ mol photons $\mathrm{m}^{-2} \mathrm{~s}^{-1} \quad\left(\mathrm{LL}_{2}\right)$, $200 \mu$ mol photons $\mathrm{m}^{-2} \quad \mathrm{~s}^{-1} \quad\left(\mathrm{LL}_{3}\right)$, and $400 \mu$ mol photons $\mathrm{m}^{-2} \mathrm{~s}^{-1}\left(\mathrm{LL}_{4}\right)$. The chlorophyll quotas corresponding to all four acclimation states are also obtained from the experimental measurements of carbon and chlorophyll content per cell reported in [25]; see data points on Figure 3. The resulting fits in terms of the chlorophyll-specific photosynthesis rate $\mu_{\mathrm{Chl}}$ are shown in Figure 2 and the corresponding parameter estimates are given in Table II. Here again, the model predictions are in good agreement with the experimental data set, thereby confirming that the 


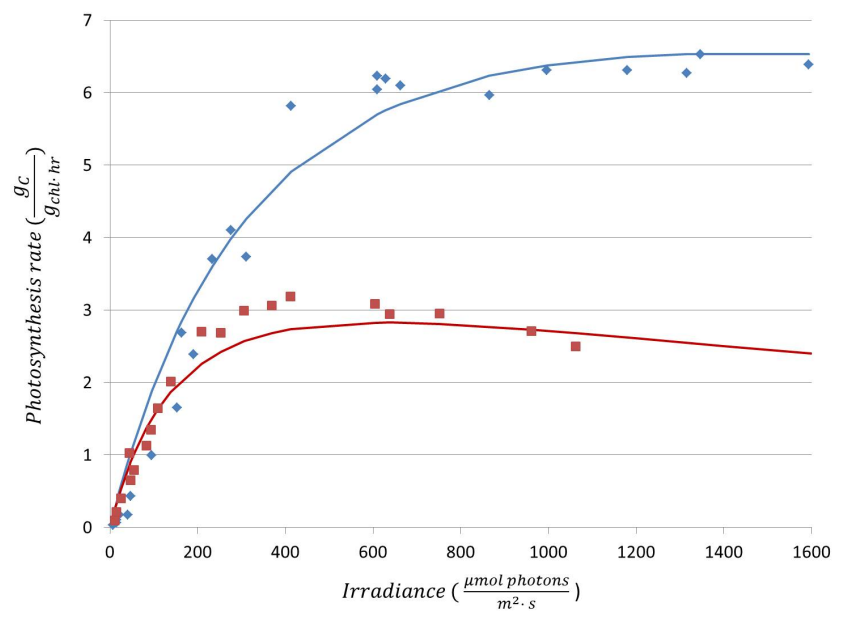

Fig. 1. Dependency between growth rate at HL (model: blue line, exp. data: blue diamonds) and LL acclimation: (model: green line, exp. data: green triangles) for Skeletonema costatum [24].

coupled model is also able to capture the photosynthetic behavior of Dunaliela tertiolocta. Nonetheless, the value of $\kappa=1.336$ does not belong to the expected range of $[0,1]$; this important aspect with be discussed further in Sect. V-B.

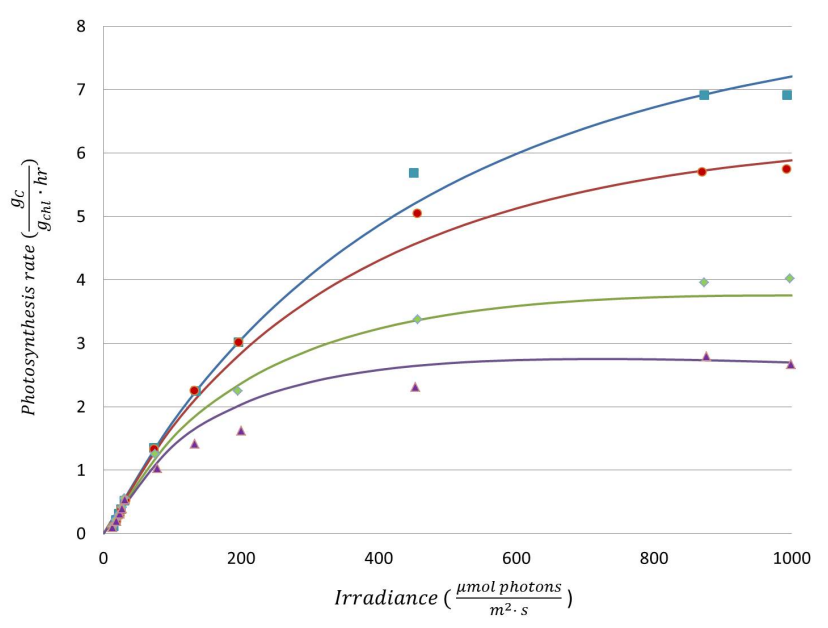

Fig. 2. Dependency between growth rate and acclimation for Dunaliela tertiolocta [25]. The blue, red, green and purple lines and points correspond to states line corresponds to $\mathrm{LL}_{4}, \mathrm{LL}_{3}, \mathrm{LL}_{2}$ and $\mathrm{LL}_{1}$ respectively.

2) Chlorophyll Content Expression: The values of the parameters $k_{I}$ and $\bar{\gamma}$ in (5) are now estimated using a nonlinear least-square minimization procedure (global optimization solver BARON in GAMS [26]).

- Experimental data from the work of Falkowski \& Owens [25] are used to calibrate the chlorophyll content expression (4),(5). The same growth irradiances as previously are considered, and the corresponding experimental measurements of the nitrogen quota $q$ and the chlorophyll content $\mathrm{Chl}$ are used. These data points are plotted in Figure 3 along with the resulting model

\begin{tabular}{ccc}
\hline Parameter & Anning et al. [24] & Falkowski \& Owens [25] \\
\hline $\bar{\mu}_{\mathrm{Chl}}$ & $0.0240 \mathrm{~g}_{\mathrm{Chl}}^{-1} \mathrm{hr}^{-1}$ & $0.0204 \mathrm{gC}_{\mathrm{Chl}}^{-1} \mathrm{hr}^{-1}$ \\
$\beta$ & 0.0030 & 0.0190 \\
$\kappa$ & 0.542 & 1.336 \\
\hline
\end{tabular}

\section{TABLE II}

ESTIMATED PARAMETERS IN THE CHLOROPHYLL-SPECIFIC PHOTOSYNTHESIS RATE (17) BASED ON THE DATA FROM ANNING ET AL. [24] AND FALKOWSKI \& OWENS [25].

fit. The estimated values for $k_{I}$ and $\bar{\gamma}$ are reported in Table III. A good agreement is observed, which provides a first validation of the chlorophyll content model (4),(5) too.

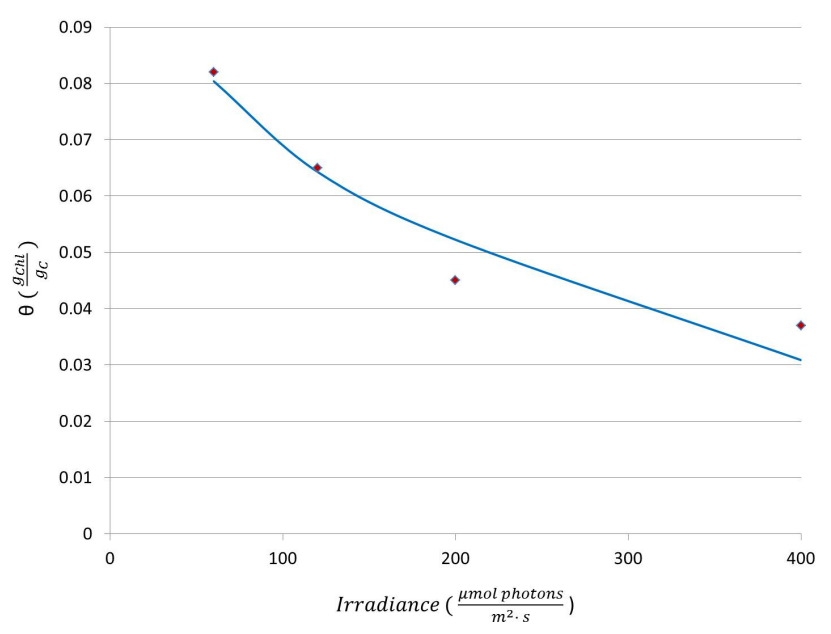

Fig. 3. Experimental data (red diamonds) and model fit (blue line) for the different acclimated states of Dunaliela tertiolocta [25].

\begin{tabular}{cc}
\hline Parameter & Value \\
\hline$k_{I}$ & $447 \mu \mathrm{mol}$ photons $\mathrm{m}^{-2} \mathrm{~s}^{-1}$ \\
$\bar{\gamma}$ & $0.310 \mathrm{gChl} \mathrm{g}_{\mathrm{N}}^{-1}$ \\
\hline & TABLE III
\end{tabular}

ESTIMATED PARAMETERS IN THE CHLOROPHYLL CONTENT (4),(5) BASED ON THE DATA FROM FALKOWSKI \& OWENS [25].

\section{B. Discussion}

The fits obtained by estimating the parameters $\beta, \kappa$ and $\bar{\mu}_{\mathrm{Chl}}$ in (17), on the one hand, and the parameters $k_{I}$ and $\bar{\gamma}$ in (4),(5), on the other hand, are in good agreement with the experimental data from Anning et al. [24] (Figure 1) and Falkowski \& Owens [25] (Figure 2). As far as the chlorophyll content model (4),(5) is concerned, it is also worth mentioning that the estimated values of $k_{I}$ and $\bar{\gamma}$ (Table III) are consistent with those reported in previous works [8].

In the photosynthesis rate model (17), the estimated value of $\kappa=0.54$ based on the data by Anning et al. [24] belongs to the expected range of $[0,1]$ per the considerations in Sect. IV-B. On the other hand, the estimated value of 
$\kappa=1.34$ from the data by Falkowski \& Owens [25] lies outside this expected range. Interestingly, this counter-intuitive relationship between $\sigma$ and $\theta$ was also pointed out by the same authors. It clearly highlights the complex underlying mechanisms to adapt quantitatively $(N)$ and qualitively $(\sigma)$ the potential of light harvesting to various light levels.

\section{CONClusions And Future Directions}

The dynamic model presented in this paper couples photosynthetic processes that act on different time scales. Photosynthetic production and inhibition act on fast time scales of seconds or minutes, while the dynamics of intracellular nitrogen and chlorophyll contents are bound to slow time scales of hours or days. The main novelty of our model lies in the use of the chlorophyll quota $\theta$ to relate the acclimation and growth processes with the states of the PSUs. A preliminary calibration and validation based on PI curve data from the literature shows encouraging results. Combined with previous (validated) models describing the dynamics of the PSUs (Han model), nitrogen content (Droop model), and chlorophyll content (Geider et al. [7] and Bernard [8]), our model is the first of its kind to simultaneously account for photoinhibition, photoacclimation and nutrient-limited growth.

The results of this work provide important insights into photoacclimation processes in microalgae. Our model builds upon the well accepted model of Han as well as widely accepted assumptions about the utilization of chlorophyll in microalgae. In order to further discriminate between the n-strategy and s-strategy of photoacclimation, more experimental data would be needed, especially data covering a wider range of acclimation states and different species. Measuring a larger set of physiological variables, such as $\sigma$ [27], would also be helpful. By making the link among different PI curves, while preserving a simple structure, the proposed model can serve as a tool to test recent hypotheses. Finally, a mathematical analysis of this model could provide valuable insight into the inherent trade-offs and eventually help identify strategies for enhancing microalgae productivity in large-scale industrial systems.

\section{ACKNOWLEDGMENT}

This work was supported by the INRIA Nautilus ARC. $\mathrm{AN}$ and BC are grateful for financial support from Marie Curie grant PCIG09-GA-2011-293953.

\section{REFERENCES}

[1] J. Sheehan, T. Dunahay, J. Benemann, and P. Roessler, “A Look Back at the U.S. Department of Energy's Aquatic Species Program Biodiesel from Algae," U.S. Department of Energy, Tech. Rep., 1998.

[2] P. J. 1. B. Williams and L. M. L. Laurens, "Microalgae as biodiesel and biomass feedstocks: Review and analysis of the biochemistry, energetics and economics," Energy \& Environmental Science, vol. 3, pp. 554-590, 2010.

[3] B. Sialve, N. Bernet, and O. Bernard, "Anaerobic digestion of microalgae as a necessary step to make microalgal biodiesel sustainable," Biotechnology Advances, vol. 27, pp. 409-416, 2009.

[4] R. H. Wijffels and M. J. Barbosa, "An outlook on microalgal biofuels," Science, vol. 329, no. 5993, pp. 796-799, 2010.

[5] F. B. Metting, "Biodiversity and application of microalgae," Journal of Industrial Microbiology \& Biotechnology, vol. 17, pp. 477-489, 1996.
[6] T. Lacour, A. Sciandra, A. Talec, P. Mayzaud, and O. Bernard, "Diel variations of carbohydrates and neutral lipids in N-sufficient and Nstarved cyclostat cultures of Isochrysis sp." Journal of Phycology, vol. 48, pp. 966-975, 2012.

[7] R. J. Geider, H. L. MacIntyre, and T. M. Kana, "Dynamic model of phytoplankton growth and acclimation: responses of the balanced growth rate and the chlorophyll $a$ :carbon ratio to light, nutrientlimitation and temperature," Marine Ecology Progress Series, vol. 148, pp. 187-200, 1997.

[8] O. Bernard, "Hurdles and challenges for modelling and control of microalgae for $\mathrm{CO}_{2}$ mitigation and biofuel production," Journal of Process Control, vol. 21, no. 10, pp. 1378-1389, 2011.

[9] B. P. Han, "Photosynthesis-irradiance response at physiological level: A mechanistic model," Journal of Theoretical Biology, vol. 213, pp. 121-127, 2001.

[10] M. R. Droop, "25 years of algal growth kinetics - A personal view," Botanica Marina, vol. 16, pp. 99-112, 1983.

[11] P. H. C. Eilers and J. C. H. Peeters, "Dynamic behavior of a model for photosynthesis and photoinhibition," Ecological Modelling, vol. 69, no. $1-2$, pp. 113-133, 1993

[12] P. G. Falkowski and J. A. Raven, Aquatic Photosynthesis, 2nd ed. Princeton University Press, 2007.

[13] A. Sciandra, "Coupling and uncoupling between nitrate uptake and growth rate in Prorocentrum minimum (Dinophyceae) under different frequencies of pulsed nitrate supply," Marine Ecology Progress Series, vol. 72, pp. 261-269, 1991.

[14] M. R. Droop, "Vitamin $B_{12}$ and marine ecology. The kinetics of uptake, growth and inhibition in Monochrysis lutheri," Journal of the Marine Biological Association of the United Kingdom, vol. 48, no. 3, pp. 689-733, 1968.

[15] K. Lange and F. J. Oyarzun, "The attractiveness of the Droop equations," Mathematical Biosciences, vol. 111, pp. 261-278, 1992.

[16] O. Bernard and J. L. Gouzé, "Transient behavior of biological loop models, with application to the Droop model," Mathematical Biosciences, vol. 127, no. 1, pp. 19-43, 1995.

[17] I. Vatcheva, H. deJong, O. Bernard, and N. J. L. Mars, "Experiment selection for the discrimination of semi-quantitative models of dynamical systems," Artificial Intelligence, vol. 170, pp. 472-506, 2006.

[18] A. Sciandra and P. Ramani, "The limitations of continuous cultures with low rates of medium renewal per cell," Journal of Experimental Marine Biology \& Ecology, vol. 178, pp. 1-15, 1994.

[19] O. Bernard and J. L. Gouzé, "Nonlinear qualitative signal processing for biological systems: application to the algal growth in bioreactors," Mathematical Biosciences, vol. 157, pp. 357-372, 1999.

[20] O. N. Ross and R. J. Geider, "New cell-based model of photosynthesis and photo-acclimation: accumulation and mobilisation of energy reserves in phytoplankton," Marine Ecology Progress Series, vol. 383, pp. 53-71, 2009.

[21] P. Hartmann, Q. Bechet, and O. Bernard, "Interaction between time scales in microalgae based processes," in 20th Mediterranean Conference on Control \& Automation (MED), July 3-6, 2012, Barcelona, Spain, 2012.

[22] H. L. MacIntyre, T. M. Kana, T. Anning, and R. J. Geider, "Photoacclimation of photosynthesis irradiance response curves and photosynthetic pigments in microlagae and cyanobacteria," Journal of Phycology, vol. 38, no. 1, pp. 17-38, 2002.

[23] K. Richardson, J. Beardall, and J. A. Raven, "Adaptation to unicellular algae to irradiance: An analysis of strategies," New Phytologist, vol. 93, pp. 157-191, 1983.

[24] T. Anning, H. L. MacIntyre, S. M. Pratt, P. J. Sammes, S. Gibb, and R. J. Geider, "Photoacclimation in the marine diatom Skeletonema costatum," Limnology \& Oceanography, vol. 45, pp. 1807-1817, 2000.

[25] P. G. Falkowski and T. G. Owens, "Light-shade adaptation: Two strategies in marine phytoplankton," Plant Physiology, vol. 66, pp. 592-595, 1980.

[26] N. V. Sahinidis and M. Tawarmalani, "GAMS-BARON Manual (ver. 11.0.2)," available at: http://www.gams.com/dd/docs/solvers/baron.pdf, Tech. Rep., June 2012.

[27] Y. Huot and M. Babin, Chlorophyll a Fluorescence in Aquatic Sciences: Methods and Applications. Dordrecht, The Netherlands: Springer, 2010. 